\title{
Approximate Analytical Solutions for the Transient Mass Loss Rate and Piloted Ignition Time of a Radiatively Heated Solid in the High Heat Flux Limit
}

\author{
CHRIS LAUTENBERGER and CARLOS FERNANDEZ-PELLO \\ Department of Mechanical Engineering \\ University of California, Berkeley \\ Berkeley, CA 94720
}

\begin{abstract}
An approximate closed form solution is developed for the mass loss rate of a semiinfinite solid irradiated by a constant net heat flux. The solution is valid at high heat flux levels where surface losses and the endothermic heat sink due to pyrolysis are small in comparison to the applied heat flux. The expression obtained for the mass loss rate is used to develop an explicit closed form relation for the time to piloted ignition using a critical mass flux as the ignition criterion. The resultant formula is identical to that obtained from the classical thermal ignition theory, with the important difference that the surface temperature at ignition is not constant. Rather, it increases with applied heat flux, mass flux at ignition, and activation temperature, and decreases with increasing density, pre-exponential factor, and thermal conductivity. The model predictions are compared to recent high-heat flux ignition measurements for PMMA.
\end{abstract}

KEYWORDS: piloted ignition, mass loss rate, in-depth pyrolysis, ignition temperature

\section{NOMENCLATURE}

\begin{tabular}{|c|c|c|c|}
\hline$a$ & constant in Eq. 9 (7.14) & $Z$ & pre-exponential factor $\left(\mathrm{s}^{-1}\right)$ \\
\hline A & constant in Eq. 10 (see Eq. 11) & & \\
\hline$b$ & constant in Eq. 9 (1.32) & \multicolumn{2}{|c|}{ Greek } \\
\hline$B$ & constant in Eq. 10 (see Eq. 11) & $\alpha$ & thermal diffusivity (m²/s) \\
\hline$C$ & specific heat $(\mathrm{kJ} / \mathrm{kg} \cdot \mathrm{K})$ & $\delta$ & thermal penetration depth (m \\
\hline C & constant - see Eq. 21 & $\rho$ & density $\left(\mathrm{kg} / \mathrm{m}^{3}\right)$ \\
\hline$\Delta H_{v}$ & heat of vaporization (J/kg) & $\mu$ & constant - see Eq. 21 \\
\hline$k$ & thermal conductivity $(\mathrm{W} / \mathrm{m} \cdot \mathrm{K})$ & $v$ & constant - see Eq. 21 \\
\hline$\ell$ & characteristic length (m) & $\xi$ & $x / \delta$ \\
\hline$L$ & sample thickness (m) & $\hat{\Lambda}$ & function - see Eq. 16 \\
\hline$\dot{m}^{\prime \prime \prime}$ & pyrolysis rate $\left(\mathrm{kg} / \mathrm{m}^{3} \cdot \mathrm{s}\right)$ & & \\
\hline$\dot{m}_{0}^{\prime \prime}$ & pyrolysis rate $\left(\mathrm{kg} / \mathrm{m}^{2} \cdot \mathrm{s}\right)$ & \multicolumn{2}{|c|}{ Subscripts } \\
\hline$\dot{q}_{e}^{\prime \prime}$ & heat flux $\left(\mathrm{W} / \mathrm{m}^{2}\right)$ & 0 & Ambient \\
\hline$t$ & time $(\mathrm{s})$ & $a$ & Activation \\
\hline$T$ & temperature (K) & ig & Ignition \\
\hline & distance into solid (m) & $r$ & Reference \\
\hline
\end{tabular}

\section{INTRODUCTION}

The classical thermal theory of piloted ignition suggests that the inverse square root of ignition time plotted against the applied heat flux should give a straight line. Indeed, ignition times measured experimentally do show the expected linear correlation at 
moderate heat flux levels $\left(<\sim 75 \mathrm{~kW} / \mathrm{m}^{2}\right)$. However, Beaulieu et al. [1] recently reported measurements made with the FM Global Advanced Flammability Measurements Apparatus (AFM) at applied heat fluxes up to $200 \mathrm{~kW} / \mathrm{m}^{2}$ that show an unexpected nonlinear trend at high heat flux levels. The observed ignition time is longer at high heat fluxes than would be expected by extrapolating ignition time data obtained at moderate heat flux levels. A similar trend is also apparent in some older measurements at high heat flux levels [2]. To the authors' knowledge, these observations have not yet been conclusively explained.

Although most fire-level heat fluxes are lower than $75 \mathrm{~kW} / \mathrm{m}^{2}$, with the notable exceptions of post-flashover burning [3] and some cases of direct flame impingement [4], there are several situations of practical interest where applied heat flux levels may be greater than $75 \mathrm{~kW} / \mathrm{m}^{2}$. Starting in the 1950's, the ignition of cellulosic materials heated by intense short duration thermal radiation characteristic of thermonuclear detonations was studied extensively, as recently summarized by Martin [5]. Perhaps more relevant to today's world, short-duration/high intensity radiant exposures may occur during electrical arcing, gaseous deflagrations, boiling liquid expanding vapor explosions (BLEVEs), hydrocarbon spray fires, and high velocity gaseous jet fires. High heat flux exposures may also occur by conductive or convective heat transfer, as in the case of ignition by molten metals (e.g., produced during electrical arcing/welding), brand spotting, etc.

In order to develop physically correct ignition models, it is important to understand the reasons that the classical thermal ignition theory under-predicts the ignition time at highheat flux levels. With this theory, ignition is assumed to occur when the surface temperature reaches a constant "ignition temperature." However, experimental measurements show that the surface temperature at ignition depends on the applied heat flux level, generally increasing with applied heat flux for polymers [6,7,8]. A more physically correct ignition criterion should include the pyrolysate mass flux since piloted ignition is a gas phase phenomenon. For this reason, another ignition criterion that has been considered is attainment of a critical mass flux rate that is sufficient to generate a combustible mixture in the vicinity of the igniter $[9,10,11]$.

It has been argued on phenomenological grounds that as the applied heat flux increases, the pyrolysis reaction is increasingly confined to a thin layer near the surface. This layer must be raised to a higher temperature to achieve the critical mass flux for ignition than at lower heat flux levels where the thermal wave penetrates deeper into the solid, involving a larger volume in the pyrolysis process [7]. The finite depth over which pyrolysis occurs causes increasingly higher surface temperatures at ignition. Therefore, as the heat flux level is increased, the thermal penetration depth has an increasingly important role in determining the total pyrolysis rate, and may become the controlling mechanism at very high heat flux levels. The purpose of this paper is to determine whether this phenomenon can explain the unexpectedly long ignition times observed at high heat flux levels [1]. Rather than resorting to numerical solution of the governing equations, a simplified closed form model that includes in-depth pyrolysis is developed to provide insight into the underlying physics.

\section{GOVERNING EQUATIONS}

In this analysis, ignition is assumed to occur when a critical mass flux rate is attained; the gas-phase induction time is inherently assumed invariant and small compared to the 
thermal time. The thermal response of the constant-property solid is governed by the onedimensional heat conduction equation with internal heat sink (due to pyrolysis):

$$
\rho c \frac{\partial T(x, t)}{\partial t}=k \frac{\partial^{2} T(x, t)}{\partial x^{2}}-\Delta H_{v} \dot{m}^{\prime \prime \prime}(x, t)
$$

The second term on the RHS is attributed to endothermic pyrolysis, which is modeled as an irreversible Arrhenius decomposition reaction:

$$
\dot{m}^{\prime \prime \prime}(x, t)=\rho Z \exp \left[-\frac{T_{a}}{T(x, t)}\right]
$$

where $Z$ is the pre-exponential factor and $T_{a}$ is the activation temperature, equal to the activation energy divided by the universal gas constant. The pyrolysis rate per unit surface area is found by evaluating the integral:

$\dot{m}_{0}^{\prime \prime}(t)=\int_{0}^{L} \dot{m}^{\prime \prime \prime}(x, t) d x=\rho Z \int_{0}^{L} \exp \left[-\frac{T_{a}}{T(x, t)}\right] d x$

where $L$ is the thickness of the solid and $x=0$ corresponds to the exposed face. The transient mass loss rate can be found by solving Eqs. 1-3 subject to appropriate initial and boundary conditions which depend on the heating mode and geometrical configuration (see e.g., Ref. [10]). In general, numerical integration is required due to the nonlinearity of the pyrolysis heat sink and radiative surface losses.

\section{APPROXIMATE SOLUTION-PYROLYSIS RATE}

In this paper, we seek to develop a closed form approximation to Eq. 3 that can be used to estimate the transient pyrolysis rate, and subsequently used to predict the ignition time using a critical mass flux as the ignition criterion. The approximate solution is made possible by:

1) assuming semi-infinite behavior (thermally thick solid, $L \rightarrow \infty$ ),

2) assuming the applied (external) heat flux is invariant and approximating the absorbed net heat flux as constant by neglecting surface heat losses,

3) assuming the solid is inert (negligible heat of vaporization),

4) fitting the analytic (exact) temperature profile obtained under approximations 1-3 with a simplified expression, and

5) replacing the Arrhenius pyrolysis rate (Eq. 2) with a power-law dependence.

Approximations 2 and 3 are justified at high heat flux levels when one considers that the total losses attributed to convection, reradiation, and endothermic pyrolysis at the ignition point are approximately equal to the "critical heat flux for ignition," of order $15 \mathrm{~kW} / \mathrm{m}^{2}$ for common polymeric and cellulosic materials. Thus, at an applied heat flux of $150 \mathrm{~kW} / \mathrm{m}^{2}$ the net heat flux decreases by $\sim 10 \%$ prior to ignition. The validity of Approximations 4 and 5 will be demonstrated below.

The solution to Eq. 1 under Approximations 1-3 above is: 
$T-T_{0}=\frac{\dot{q}_{e}^{\prime \prime}}{k}\left[\frac{\delta}{\sqrt{\pi}} \exp \left(-\left(\frac{x}{\delta}\right)^{2}\right)-x \operatorname{erfc}\left(\frac{x}{\delta}\right)\right]$

In Eq. $4, \delta$ is the thermal penetration depth, defined as:

$\delta=\sqrt{4 \alpha t}$

After introducing the dimensionless distance $\xi=x / \delta$, Eq. 4 becomes:

$\frac{T-T_{0}}{\delta \dot{q}_{e}^{\prime \prime} / k}=\left[\frac{\exp \left(-\xi^{2}\right)}{\sqrt{\pi}}-\xi \operatorname{erfc}(\xi)\right]$

It can now be seen from Eq. 6 that the temperature profile is invariant with time when presented as a function of distance into the solid normalized by the thermal penetration depth. The entire temperature profile is "stretched" by $\delta$, i.e., it grows proportional to the square root of time. The pyrolysis rate per unit surface area can be calculated from Eq. 3:

$\dot{m}_{0}^{\prime \prime}=\rho Z \delta \int_{0}^{\infty} \exp \left(-\frac{T_{a}}{T}\right) d \xi \approx \rho Z \delta \int_{0}^{1} \exp \left(-\frac{T_{a}}{T}\right) d \xi$

where the independent variable has been changed from $x$ to $\xi$. The upper limit of integration can be changed from $\infty$ to 1 because prior to ignition, the pyrolysis occurs at depths smaller than the thermal penetration depth.

Substituting Eq. 6 into Eq. 7, the following expression for the pyrolysis rate is obtained:

$\frac{\dot{m}_{0}^{\prime \prime}}{\rho Z \delta}=\int_{0}^{1} \exp \left(-\frac{T_{a}}{T_{0}+\frac{\delta \dot{q}_{e}^{\prime \prime}}{k}\left[\frac{\exp \left(-\xi^{2}\right)}{\sqrt{\pi}}-\xi \operatorname{erfc}(\xi)\right]}\right) d \xi$

The integral on the RHS cannot be evaluated analytically. A closed form solution can be obtained by invoking Approximations 4 and 5 above. First, the temperature profile is approximated as:

$T_{0}+\frac{\delta \dot{q}_{e}^{\prime \prime}}{k}\left[\frac{\exp \left(-\xi^{2}\right)}{\sqrt{\pi}}-\xi \operatorname{erfc}(\xi)\right] \approx T_{0}+\frac{\delta \dot{q}_{e}^{\prime \prime} / k}{\sqrt{\pi}+a \xi^{b}}$

The constants $a \approx 7.14$, and $b \approx 1.32$ were found to give a good approximation to the exact profile for $0<\xi<0.4$, the region with the largest contribution to the pyrolysis rate. The approximation $\left(\exp \left(-\xi^{2}\right) / \sqrt{\pi}-\xi \operatorname{erfc}(\xi) \approx\left(\sqrt{\pi}+a \xi^{b}\right)^{-1}\right.$ ) is shown in Fig. 1 . 


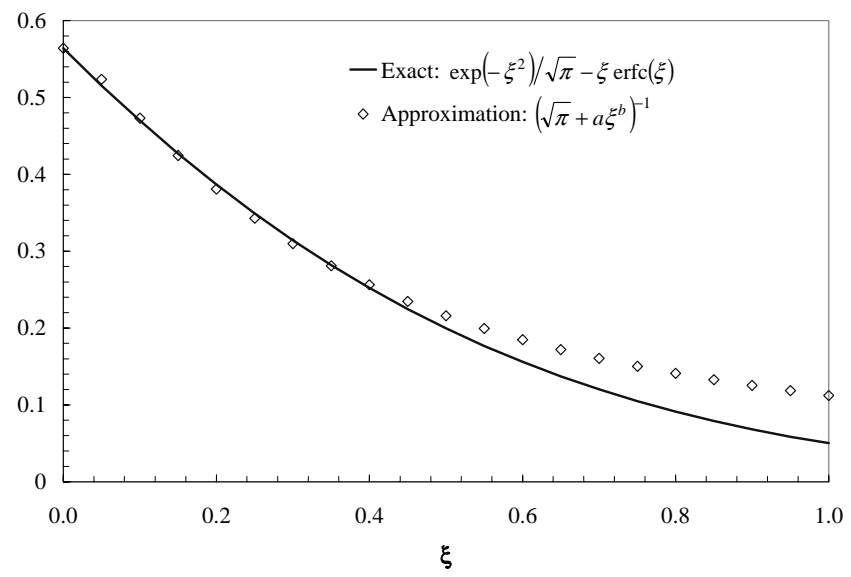

Fig. 1. Approximation to temperature profile.

The final approximation involves replacing the Arrhenius pyrolysis rate with a power law function:

$\exp \left(-\frac{T_{a}}{T}\right) \approx A\left(\frac{T}{T_{r}}\right)^{B}$

where $T_{r}$ is a reference temperature (taken here as equal to the initial temperature, $T_{0}=$ $300 \mathrm{~K})$. Alternatively, a Taylor series could also be used, but the power law form is selected here to avoid summing over multiple terms. The constants $A$ and $B$ depend on the activation temperature of the material under consideration. The functional form of $A$ and $B$ found to minimize the error in Eq. 10 over the temperature range $250^{\circ} \mathrm{C}<T<$ $450^{\circ} \mathrm{C}$ are:

$A=\exp \left(-T_{a} / T_{1}\right) ; T_{1} \approx 357 \mathrm{~K}$

$B=T_{a} / T_{2} ; T_{2} \approx 615 \mathrm{~K}$

This temperature range was selected because the surface temperature at piloted ignition is generally between $250^{\circ} \mathrm{C}$ and $450^{\circ} \mathrm{C}$. The Arrhenius function and its power-law approximation (Eq. 10) are shown in Fig. 2 for activation temperatures ranging from $10,000 \mathrm{~K}(83 \mathrm{~kJ} / \mathrm{mol})$ to $30,000 \mathrm{~K}(250 \mathrm{~kJ} / \mathrm{mol})$. 


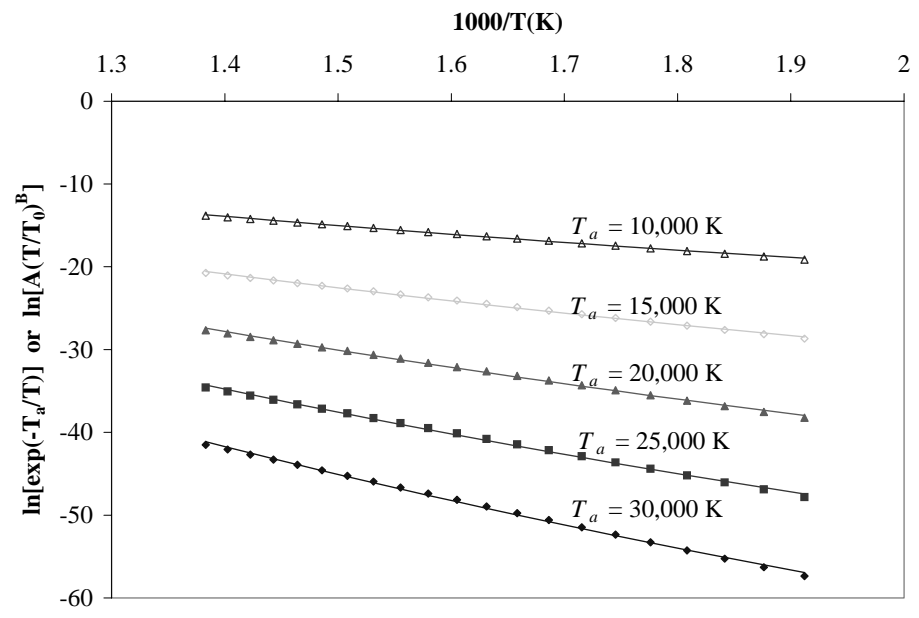

Fig. 2. Power law approximation to Arrhenius function. Individual points are Arrhenius function and solid lines are power law approximation. See Eqs. 10-11.

After substituting Eq. 9 and Eq. 10 into Eq. 8 (or equivalently, Eqs. 6, 9, and 10 into Eq. 7), the pyrolysis rate can be calculated from the integral:

$$
\frac{\dot{m}_{0}^{\prime \prime}}{\rho Z \delta} \approx \frac{A}{T_{0}^{B}} \int_{0}^{1}\left(T_{0}+\frac{\delta \dot{q}_{e}^{\prime \prime} / k}{\sqrt{\pi}+a \xi^{b}}\right)^{B} d \xi
$$

The exact solution to Eq. 12 is:

$$
\frac{\dot{m}_{0}^{\prime \prime}}{\rho A Z \delta}=\left(1+\frac{\delta / \ell}{\sqrt{\pi}}\right)^{B} f_{1}(\delta / \ell)
$$

where $\ell=k T_{0} / \dot{q}_{e}^{\prime \prime}$ is a characteristic length scale related to the thickness of the layer with high temperature gradients and $f_{1}(\delta / \ell)$ is Appell's $F_{1}$ hypergeometric function [12]:

$$
f_{1}(\delta / \ell) \equiv F_{1}\left(\frac{1}{b}, B,-B, 1+\frac{1}{b},-\frac{a}{\sqrt{\pi}},-\frac{a}{\sqrt{\pi}+\delta / \ell}\right)
$$

It is useful to multiply both sides of Eq. 13 by $\delta / \ell$, giving:

$$
\frac{\dot{m}_{0}^{\prime \prime}}{\rho A Z \ell}=\left(1+\frac{\delta / \ell}{\sqrt{\pi}}\right)^{B} \frac{\delta}{\ell} f_{1}\left(\frac{\delta}{\ell}\right)
$$

Since $B$ is generally $>15$, the first term on the RHS of Eq. 15 (in parentheses) dominates the pyrolysis rate if $(\delta / \ell) f_{1}(\delta / \ell)$ is not a steep function of $\delta / \ell$. It was found that for $\delta / \ell>1$, the first term on the RHS of Eq. 15 is at least four orders of magnitude greater than $(\delta / \ell) f_{1}(\delta / \ell)$, and that the function $(\delta / \ell) f_{1}(\delta / \ell)$ can be approximated by a simple linear function $\Lambda$ : 
$\Lambda\left(\frac{\delta}{\ell}\right) \equiv \frac{\delta}{\ell} f_{1}\left(\frac{\delta}{\ell}\right) \approx \frac{1000}{T_{a}^{1.04}}+\frac{26}{T_{a}^{0.71}} \frac{\delta}{\ell}$

Figure 3 shows the variation of $(\delta / \ell) f_{1}(\delta / \ell)$ with $\delta / \ell$ for several values of $T_{a}$, as well as the approximation $\Lambda$ given in Eq. 16. Appell's $F_{1}$ hypergeometric function was evaluated with a numerical code by Colavecchia [12].

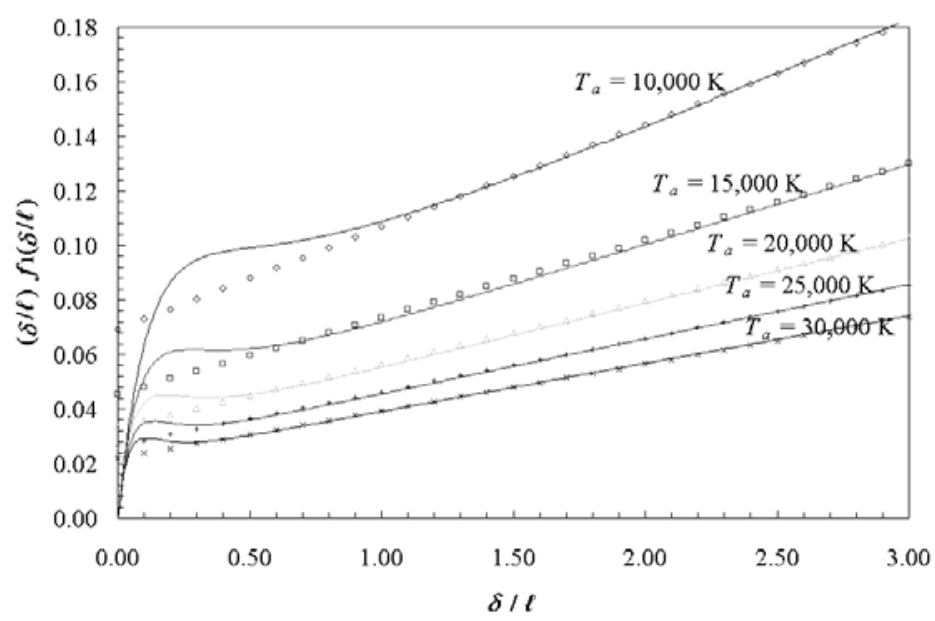

Fig. 3. Variation of $(\delta / \ell) f_{1}(\delta / \ell)$ with $\delta / \ell$ for several values of $T_{a}$. Solid lines are exact function and individual points are the linear approximation (Eq. 16).

It can be seen that Eq. 16 is a good fit to $(\delta / \ell) f_{1}(\delta / \ell)$ only for $\delta / \ell>1$ (except at higher activation temperatures where it is a good fit for $\delta / \ell>\sim 0.5$ ). However, this should have a minor effect on the calculated pyrolysis rate because the power law term in Eq. 15 is dominant. Additionally, $\delta / \ell<1$ generally corresponds to the inert heating regime where the pyrolysis rate is negligible.

The final form of the approximate expression for the transient pyrolysis rate is:

$\dot{m}_{0}^{\prime \prime}=\rho Z \ell \Lambda \exp \left(-\frac{T_{a}}{T_{1}}\right)\left(1+\frac{\delta / \ell}{\sqrt{\pi}}\right)^{\frac{T_{a}}{T_{2}}}$

where $\delta=\sqrt{4 \alpha t}, \ell=k T_{0} / \dot{q}_{e}^{\prime \prime}, T_{1}$ and $T_{2}$ are given in Eq. 11, and the function $\Lambda$ is given by Eq. 16. A spreadsheet implementation of Eq. 17 can used to estimate the transient mass loss rate in the high heat flux limit as a function of the relevant parameters. The independent variable $\delta / \ell$ in Eq. 17 is the square root of dimensionless time:

$\frac{\delta}{\ell} \equiv \sqrt{\frac{t}{t_{c}}}$ where $t_{c}=\frac{k \rho c T_{0}^{2}}{4 \dot{q}_{e}^{\prime \prime 2}}$

Here, $t_{c}$ is associated with the time required for the surface temperature to reach a certain value. Figure 4 gives a comparison of the pyrolysis rate predicted by Eq. 17 as well as the "exact" solution (Eq. 15) and the numerical solution to Eqs. 1-3 with no heat losses and $\Delta H_{v}=0$. It can be seen from Fig. 4 that both Eq. 15 and Eq. 17 match the numerical 
solution very well. The properties used in the calculation are identical to those used by Staggs [13] for black PMMA, namely: $k=0.22 \mathrm{~W} / \mathrm{m} \mathrm{K}, \rho=1190 \mathrm{~kg} / \mathrm{m}^{3}, c=1420 \mathrm{~J} / \mathrm{kg}-$ $\mathrm{K}, Z=1.0226 \times 10^{6} \mathrm{~s}^{-1}$, and $T_{a}=11,670 \mathrm{~K}$. The calculations were performed for heat flux levels of $150 \mathrm{~kW} / \mathrm{m}^{2}$ and $200 \mathrm{~kW} / \mathrm{m}^{2}$.

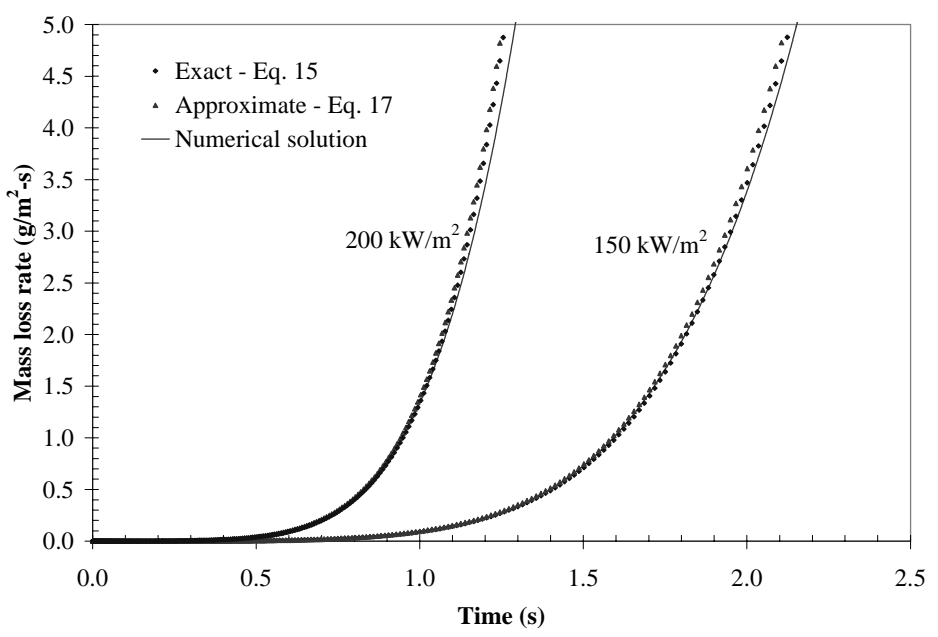

Fig. 4. Comparison of approximate solutions and numerical solution for transient pyrolysis rate.

\section{APPROXIMATE SOLUTION-TIME TO PILOTED IGNITION}

Although Eq. 17 can be solved iteratively (implicitly) for $t_{i g}$, an explicit expression for the time to ignition can be derived from Eq. 17 if the function $\Lambda$ is replaced with a constant $C$. The value of $\delta / \ell$ at which $C$ is evaluated (see Fig. 3) is not crucial when using a critical mass flux as the ignition criterion since the power law term in Eq. 17 dominates the pyrolysis rate. In order to obtain an explicit expression for the ignition time based on attainment of a critical mass flux, the classical thermal ignition theory based on a constant ignition temperature is used to estimate the value of $\delta / \ell$ at which $C$ should be evaluated:

$\sqrt{t_{i g}} \approx \sqrt{\frac{\pi}{4} k \rho c} \frac{T_{i g}-T_{0}}{\dot{q}_{e}^{\prime \prime}}$

After substituting Eq. 19 into the definition of $\delta_{\text {ig }} / \ell$ :

$\frac{\delta_{i g}}{\ell}=\frac{\sqrt{4 \alpha t_{i g}}}{k T_{0} / \dot{q}_{e}^{\prime \prime}}=\frac{\dot{q}_{e}^{\prime \prime} \sqrt{4 \alpha}}{k T_{0}} \sqrt{\frac{\pi}{4} k \rho c} \frac{T_{i g}-T_{0}}{\dot{q}_{e}^{\prime \prime}}=\sqrt{\pi} \frac{\left(T_{i g}-T_{0}\right)}{T_{0}}$

we see that $\delta_{i g} / \ell$ is approximately 2 by evaluating the rightmost term in Eq. 20 with typical values of $T_{i g}\left(300^{\circ} \mathrm{C}-400^{\circ} \mathrm{C}\right)$. The following expression was found to give a good fit to $(\delta / \ell) f_{1}(\delta / \ell)$ evaluated at $\delta / \ell=2$ : 
$C \approx \Lambda\left(\frac{\delta_{i g}}{\ell}\right) \approx \mu / T_{a}^{v} ; \mu \approx 341.3 ; \quad v \approx 0.85$

After replacing the function $\Lambda$ by the constant $C$ and substituting $\delta=\delta_{i g}$ and $\dot{m}_{0}^{\prime \prime}=\dot{m}_{i g}^{\prime \prime}$,

Eq. 17 simplifies to:

$\dot{m}_{i g}^{\prime \prime}=\rho Z \ell \Lambda\left(\frac{\delta_{i g}}{\ell}\right) \exp \left(-\frac{T_{a}}{T_{1}}\right)\left(1+\frac{\delta_{i g} / \ell}{\sqrt{\pi}}\right)^{\frac{T_{a}}{T_{2}}} \approx \rho Z \ell C \exp \left(-\frac{T_{a}}{T_{1}}\right)\left(1+\frac{\delta_{i g} / \ell}{\sqrt{\pi}}\right)^{\frac{T_{a}}{T_{2}}}$

Eq. 22 can be solved for $\delta_{i g} / \ell$ :

$$
\frac{\delta_{i g}}{\ell}=\sqrt{\pi}\left(\left(\frac{\dot{m}_{i g}^{\prime \prime}}{C \rho \exp \left(-T_{a} / T_{1}\right) Z \ell}\right)^{T_{2} / T_{a}}-1\right)
$$

Substituting $\delta_{i g}=\sqrt{4 \alpha t_{i g}}$ and $\ell=k T_{0} / \dot{q}_{e}^{\prime \prime}$ into Eq. 23, and solving for $\sqrt{t_{i g}}$ :

$$
\sqrt{t_{i g}}=\sqrt{\frac{\pi}{4} k \rho c} \frac{T_{0}}{\dot{q}_{e}^{\prime \prime}}\left(\left(\frac{\dot{q}_{e}^{\prime \prime} \dot{m}_{i g}^{\prime \prime}}{C \rho \exp \left(-T_{a} / T_{1}\right) Z k T_{0}}\right)^{T_{2} / T_{a}}-1\right)
$$

Eq. 24 can be rewritten as:

$$
\sqrt{t_{i g}}=\sqrt{\frac{\pi}{4} k \rho c} \frac{T_{i g}^{*}-T_{0}}{\dot{q}_{e}^{\prime \prime}}
$$

where

$$
T_{i g}^{*}=T_{0}\left(\frac{T_{a}^{v} \dot{q}_{e}^{\prime \prime} \dot{m}_{i g}^{\prime \prime}}{k \rho \mu Z T_{0} \exp \left(-T_{a} / T_{1}\right)}\right)^{T_{2} / T_{a}}
$$

Note that Eq. 25 is identical to the expression obtained from the thermal ignition theory (i.e., Eq. 19). However, the ignition temperature in Eq. 25 is not constant, but rather given by Eq. 26 (the values of $T_{1}, T_{2}, \mu$, and $v$ are given in Eq. 11 and Eq. 21).

\section{RESULTS}

Figure 5 gives a comparison of the ignition time calculated with the model developed above and experimental data for $25 \mathrm{~mm}$ black PMMA tested in the FM FPA, Cone Calorimeter, and FM AFM [1,14,15]. The same thermophysical properties used to generate Fig. 4 are also used in Fig. 5. Although the critical mass flux for ignition is expected to vary slightly between different fire tests, it was assumed to be $3 \mathrm{~g} / \mathrm{m}^{2}-\mathrm{s}$, independent of apparatus. This is consistent with the critical mass flux for ignition measured in the Cone Calorimeter for PMMA [16]. 


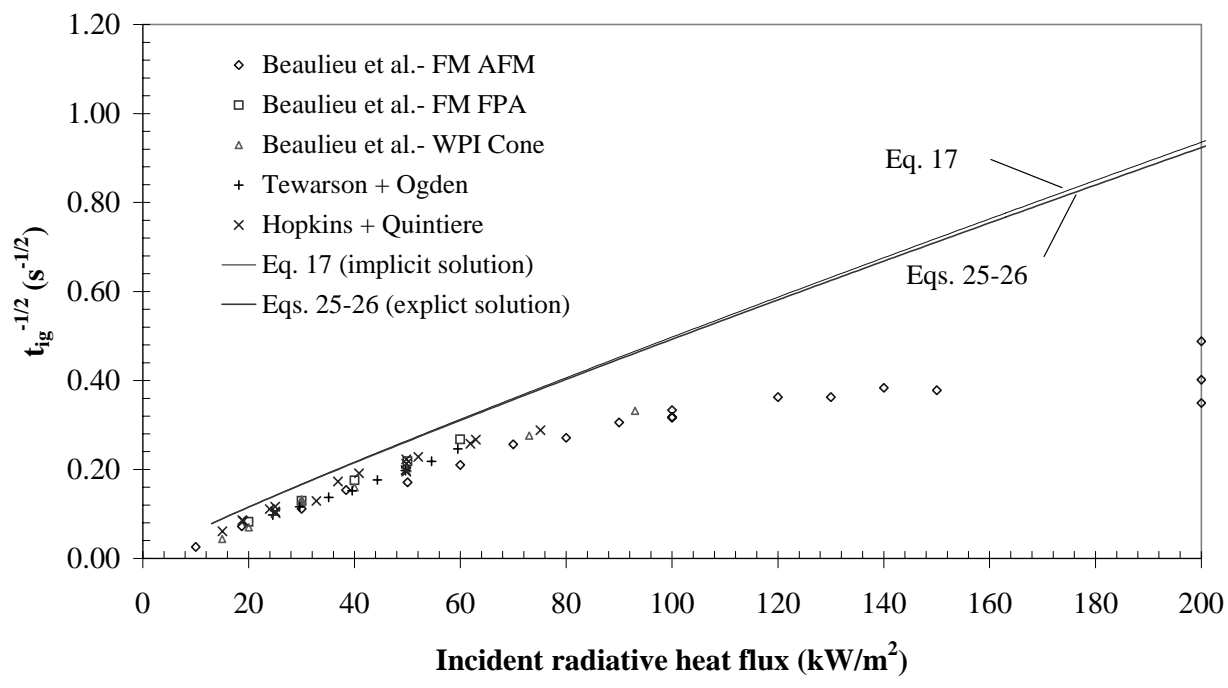

Fig. 5. Ignition time calculated with Eq. 17 and

Eqs. 25-26 compared with experimental data.

In Fig. 5, the ignition time was calculated in two ways: implicitly with Eq. 17 and explicitly with Eqs. 25 and 26. Recall that the explicit solution (Eqs. 25 and 26) was obtained by replacing $\delta_{i g} / \ell$ in Eq. 16 with a constant, i.e., by eliminating the dependency of $\Lambda$ on $\delta_{i g} / \ell$ (see Eq. 21). However, this approximation is not necessary if Eq. 17 is solved implicitly for $\delta_{i g} / \ell$. Figure 5 shows that that the ignition time calculated with the approximate explicit method is very close to that calculated with the implicit method. The reason for this is that the power law term in Eq. 17 dominates the calculated pyrolysis rate.

It can be seen from Fig. 5 that the time to ignition is underpredicted. This is expected since surface heat losses have been neglected. However, the model does not capture the nonlinear ignition time trends [1] discussed earlier. The reason for this is that the exponent $T_{2} / T_{a}$ is approximately 0.05 . Thus, if the heat flux is doubled, the surface temperature at ignition increases by less than $4 \%$. Although the mass flux at ignition is generally reported to increase slightly with applied heat flux, e.g., Panagiotou and Quintiere [16], this variation is probably not strong enough to explain the unexpected nonlinear trends.

\section{CONCLUSION}

Simple approximate closed form expressions for the transient pyrolysis rate and piloted ignition time of a semi-infinite solid have been developed. The primary weakness of the analysis is that heat sink due to endothermic gasification and the convective/reradiative surface losses are not considered. Nonetheless, these approximations provide conservative estimates of the mass loss rate and ignition time at high heat flux levels.

Although the model predicts an increase in the ignition temperature with the applied heat flux level, this variation is not strong enough to explain the unexpectedly long ignition times observed at high heat flux levels [1]. Apparently, the simple analysis presented here 
does not account for some physics of the problem that become important at high heat flux levels. Possible phenomenological explanations for the experimental observations include:

1) A shift in the spectral distribution of the applied radiation toward shorter wavelengths at high heat flux levels due to the increase in heater filament temperature in the FPA and AFM apparatus (Wien's displacement law) resulting in reduced radiative absorptivity or increased diathermancy; it is not clear how important this effect is since the black PMMA samples were coated with carbon black in the AFM and FPA;

2) increasing relative importance of gas-phase transport and induction time (i.e., the time required for sustained ignition to occur once a 'flammable' mixture forms in the vicinity of the igniter) compared to solid-phase heating and pyrolysis time;

3) rapid formation of a thin gasification layer near the surface (or a change in thermophysical properties in the thin surface layer due to temperature-dependent thermal conductivity, specific heat, density) that reduces heat transfer to the interior of the solid; and

4) a shift in the composition of the gasification products, perhaps attributed to increased oxidative pyrolysis in the thin oxygen-affected layer resulting in enhanced dilution of the flammable pyrolysate by $\mathrm{CO}$ and $\mathrm{CO}_{2}$, causing the critical mass flux for ignition to increase with applied heat flux.

It may be possible to more accurately predict the observed high heat flux ignition data with a numerical model that explicitly considers these phenomena. Item 4 may partially explain the observed increase in critical mass flux for ignition with applied heat flux [16], but the effect is probably too weak to explain the unexpectedly long ignition times at high heat flux levels [1]. Additionally, it is possible that use of a single Arrhenius reaction to describe the pyrolysis rate may not be adequate, either due to the existence of two pyrolysis regimes [17] or an analog to the negative coefficient of reaction rate used to explain long induction times in ignition of gaseous hydrocarbon fuels [18].

\section{ACKNOWLEDGEMENTS}

The authors would like to thank Patricia Beaulieu at FM Global for providing the experimental data shown in Fig. 5 and Guillermo Rein for reading this paper and offering valuable comments. The first author would like to thank NASA Glenn Research Center for support under the Graduate Student Researcher Program Grant no. NNC-04HA08H.

\section{REFERENCES}

[1] Beaulieu, P.A., Dembsey, N.A., and Alpert, R.L., "Flammability Characteristics at Applied Heat Flux Levels Up to $200 \mathrm{~kW} / \mathrm{m}^{2}$ : Time to Ignition and Mass Loss Flux,” Interflam 2004, Interscience Communications Ltd., 2004, pp. 917-928.

[2] Mikkola, E., and Wichman, I.S., "On the Thermal Ignition of Combustible Materials,” Fire and Materials, 14, pp. 87-96, (1989).

[3] Fang, J.B., and Breese, J.N., "Fire Development in Residential Basement Room,” National Bureau of Standards Report NBSIR 80-2120, Gaithersburg, MD, 1980.

[4] Lattimer, B.Y., "Heat Fluxes from Fires to Surfaces," The SFPE Handbook of Fire Protection Engineering ( $3^{\text {rd }}$ ed), DiNenno P.J. (ed.), National Fire Protection Association, Quincy, MA 02269, 2002, p. 2/14. 
[5] Martin, S.B, "Fire Setting by Nuclear Explosion: A Revisit and Use in Nonnuclear Applications," Journal of Fire Protection Engineering, 14, pp. 283297, (2004).

[6] Thomson, H.E., Drysdale, D.D., and Beyler, C.L., "An Experimental Evaluation of Critical Surface Temperature as a Criterion for Piloted Ignition of Solid Fuels,” Fire Safety Journal, 13, pp. 185-196, (1988).

[7] Cordova, J.L., Walther, D.C., Torero J.L., and Ferndandez-Pello, A.C., "Oxidizer Flow Effects on the Flammability of Solid Combustibles," Combustion Science and Technology, 164, pp. 253-278, (2001).

[8] Stevanovic, A., Mehta, S., Walther, D.C., and Fernandez-Pello, A.C., "The Effect of Fiberglass Concentration on the Piloted Ignition of Polypropylene/ fiberglass Composites,” Combustion Science and Technology, 174, pp. 171-186, (2002).

[9] Rasbash, D.J., Drysdale D.D., and Deepak, D., "Critical Heat and Mass Transfer at Pilot Ignition and Extinction of a Material," Fire Safety Journal, 10, pp. 1-10, (1986).

[10] Atreya, A. "Ignition of Fires," Philosophical Transactions of the Royal Society of London, 356, pp. 2787-2813, (1998).

[11] Zhou, Y.Y., Walther, D.C., and Fernandez-Pello A.C., "Numerical Analysis of Piloted Ignition of Polymeric Materials," Combustion and Flame, 131, pp. 147158, (2002).

[12] Colavecchia, F.D., and Gasaneoc, G., "F1: A Code to Compute Appell's $F_{1}$ Hypergeometric Function,” Computer Physics Communications, 157, pp. 32-38, (2004).

[13] Staggs, J.E.J., "The Heat of Gasification of Polymers,"Fire Safety Journal 39, pp. 711-720, (2004).

[14] Tewarson, A. and Ogden, S., "Fire Behavior of Polymethylmethacrylate," Combustion and Flame, 89, pp. 237-259, (1992).

[15] Hopkins, D. and Quintiere, J., "Material Fire Properties and Predictions for Thermoplastics,” Fire Safety Journal, 26, pp. 241-268, (1996).

[16] Panagiotou, J., and Quintiere, J.G., "Generalizing Flammability of Materials," Interflam 2004, Interscience Communications Ltd., 2004, pp. 895-905.

[17] di Blasi, C., "Modeling and Simulation of Combustion Processes of Charring and Non-charring Solid Fuels," Progress in Energy and Combustion Science, 19, pp. 71-104, (1993).

[18] Glassman, I., Combustion, Second Edition. Academic Press, Orlando, 1987, p. 73. 